\title{
Autophagy Genes (ATG16L1/IRGM) and IBD: Involvement of the ATG16L1 Gene in Algerian Patients with Crohn's Disease \\ Type of article: conference abstract
}

\author{
Aziza Boukercha ${ }^{1,2}$, Hamida Mesbah-Amroun ${ }^{1}$, Amira Bouzidi $^{1}$, Houria Saoula $^{3}$, \\ MhamedNakkemouche ${ }^{3}$, Maryline Roy ${ }^{4}$, Jean-Pierre Hugot ${ }^{4,5}$, Chafia Touil- \\ Boukoffa ${ }^{1}$. \\ ${ }^{1}$ Team Cytokines and NO Synthases, Laboratory of Cellular and Molecular Biology, \\ Faculty of Biological Sciences, University of Sciences and Technology \\ HouariBoumediene, Algiers, Algeria \\ ${ }^{2}$ University M'Hamed Bougara of Boumerdes, Faculty of Biological Sciences, \\ Algeria \\ ${ }^{3}$ Department of Gastroenterology, Maillot University Hospital, Algiers, Algeria \\ ${ }^{4}$ Team Intestinal Inflammation, INSERM UMR1149, Xavier Bichat Faculty, Paris \\ Diderot University, France \\ ${ }^{5}$ Department of Gastroenterology, Robert Debré University Hospital, France \\ Corresponding Author: boukercha.aziza@gmail.com
}

\begin{abstract}
:
Background: Chronic Inflammatory Bowel Diseases (IBD) including Crohn's disease (CD) and ulcerative colitis (UC) are gastrointestinal disorders under the influence of a complex genetic basis. One hundred sixty-three predisposition loci were identified by genome-wide association (GWAS) studies, refocusing the pathogenesis of IBD on immunity genes. Autophagy is a fundamental mechanism in the maintenance of intestinal homeostasis. Failures of this mechanism appear to be a major risk factor in the setting up of chronic intestinal inflammation. Two single nucleotide polymorphisms (SNPs) within two genes, were selected namely (rs2241880 A $\rightarrow \mathrm{G}$ ) for ATG16L1 and (rs10065172 $\mathrm{C} \rightarrow \mathrm{T}$ ) for IRGM.These two genes are both involved in the autophagy mechanism. We investigated these two SNP in an Algerian cohort for the first time since no previous association studies between IBD and the two studied genes were available for the Algerian population.
\end{abstract}

Methods: A case-control study was performed on a cohort including 95 Algerian patients with Crohn's disease and ulcerative colitis versus 116 Controls. Genotyping of the cohort involved allelic discrimination by TaqMan SNP Genotyping Assay.

Results: A statistically significant association of the rs2241880 A $\rightarrow$ Gmutation of the ATG16L1 gene was demonstrated in IBD $(p=0.04)$ and, more particularly in Crohn's disease $(p=0.03)$. The presence of this mutation would increase the risk of Crohn's disease by a factor of 2 in its GG homozygous mutated form $(\mathrm{OR}=2.1195 \% \mathrm{CI}$ (1.07- 
4.16)). No significant associations were found for the rs10065172 $\mathrm{C} \rightarrow \mathrm{T}$ mutation of the IRGM gene.

Conclusion: Our data highlight the involvement of the ATG16L1 gene in the genesis and/or evolution of IBD cases, notably Crohn's disease, indicating the impact that deregulation of the autophagy mechanism could have on the Algerian population. Nevertheless, it would be interesting to expand the sampling and carry out the functional studies to elucidate the impact of the deregulation of the ATG16L1 gene in IBD.

Keywords: Chronic Inflammatory Bowel Diseases, IBD, PCR-RFLP, SNP, ATG16L1, IRGM, Crohn's Disease,

\section{Declaration of conflicts}

This article is a conference abstract selected from the abstract book of the International Congress on Health Sciences and Medical Technologies ICHSMT'19, Tlemcen, Algeria, December 05-07, 2019.

\section{Authors' Biography}

No biography

\section{References}

No references 